\title{
Thionolactone as Resin Additive to Prepare (bio)degradable 3D Objects via VAT Photopolymerization
}

\author{
Noémie Gil, ${ }^{[a], \ddagger}$ Constance Thomas, ${ }^{[b],[c], \pm}$ Rana Mhanna, ${ }^{[b],[c]}$ Jessica Mauriello, ${ }^{[a]}$ Romain Maury, ${ }^{[a]}$ \\ Benjamin Leuschel, ${ }^{[b],[c]}$ Jean-Pierre Malval, ${ }^{[b],[c]}$ Jean-Louis Clément, ${ }^{[a]}$ Didier Gigmes, ${ }^{[a]}$ Catherine \\ Lefay, ${ }^{[\mathrm{a}]}$ Olivier Soppera ${ }^{[\mathrm{b}],[\mathrm{cc}], *}$ and Yohann Guillaneuf ${ }^{[\mathrm{a}], *}$
}

[a] N. Gil, J. Mauriello, R. Maury, Dr. J.-L. Clément, Dr. D. Gigmes, Dr. C. Lefay, Dr. Y. Guillaneuf

Aix-Marseille Univ., CNRS, Institut de Chimie Radicalaire (UMR 7273),

Av. Esc. Normendie-Niemen, Case 542, 13397 Cedex 20, France

E-mail: yohann.guillaneuf@univ-amu.fr

[b] C. Thomas, Dr. R. X, B. Leuschel, Dr. J.-P. Malval, Dr. O. Soppera

Université de Haute-Alsace CNRS, IS2M UMR 7361

Mulhouse 68100, France

E-mail: Olivier.soppera@uha.fr

[c] C. Thomas, Dr. R. X, B. Leuschel, Dr. J.-P. Malval, Dr. O. Soppera

Université de Strasbourg, Strasbourg, France

¥ The two authors contributed equally

Supporting information for this article is given via a link at the end of the document.

\begin{abstract}
D printing and especially VAT photopolymerization leads to cross-linked materials with high thermal, chemical and mechanical properties. Nevertheless, such stability is incompatible with degradability and re/upcyclability. We showed here that thionolactone and especially dibenzo[c,e]-oxepane-5-thione (DOT) could be used as an additive (2 wt $\%$ ) to acrylate-based resins to introduce weak bonds into the network via a radical ring-opening polymerization process. The low amount of additive allows to only slightly modify the printability of the resin, keep intact its resolution and maintain the mechanical properties of the 3D object. The resin with additive was used in UV microfabrication and 2-photon stereolithography setup and commercial $3 \mathrm{D}$ printers. The fabricated objects were shown to degrade in basic solvent as well in a home-made compost. The rate of degradation is nonetheless dependent of the size of the object. This feature was used to prepare 3D objects with support structures that could be easily solubilized.
\end{abstract}

\section{Introduction}

Additive manufacturing and more specially $3 \mathrm{D}$ printing has revolutionized the classic industrial manufacturing by creating directly a 3D object that was designed on a computer using successive layering of materials. ${ }^{[1]}$ The rapid development of this technique was first made by the ease of the fused deposition modeling (FDM) technique that allows to prepare 3D objects by the computer-controlled layer by layer deposition of molten or semi-molten polymer via an extrusion nozzle. ${ }^{[1]}$ Nevertheless, 3D printing has become extremely popular with the development of light-cured processes that are summarized by the term VAT photopolymerization. ${ }^{[1]}$ Among these processes, Digital Light Processing (DLP) ${ }^{[1-2]}$ in particular has attracted attention due to several attractive features, such as fast build, low resolution $(<100$ $\mu \mathrm{m}$ features), wide ink viscosity tolerance and low cost. The 3D object is thus obtained by the cross-linking of a liquid resin consisting mainly of multifunctional (meth)acrylates derivatives. The photopolymerization of acrylates occurs rapidly, with high yields, and generates almost no byproducts. This led to a crosslinked material whose mechanical properties could be tuned by the monomers structure. Moreover, such object is constituted by a $\mathrm{C}-\mathrm{C}$ bond covalent network that impart a high thermal and chemical stability. This permanent cross-linked network could also be detrimental for diverse reasons. First the high stability is incompatible with facile degradability and re/upcyclability. The high volume/amount of 3D-printed thermoset will be incinerated or stored in landfills following use, making this so promising manufacturing process not environment-friendly or sustainable. ${ }^{[3]}$ Secondly, this high stability is also not compatible with subtractive manufacturing, ${ }^{[4]}$ that is a technique where printed materials could be removable or erasable after its formation. Such approach ${ }^{[5]}$ is very interesting to easily remove templates or sacrificial parts of $3 \mathrm{D}$ objects, a technique that is widely used when the object is a positive print before casting and lastly decomposed such as in jewelry or to prepare ceramics. Recently very elegant approaches were proposed by Barner-Kowollik and coworkers that developed new monomers and resins based either on thiol-ene chemistry, ${ }^{[6]}$ TAD-polyether ligation, ${ }^{[7]}$ silane-based monomers, ${ }^{[8]}$ o-nitrobenzyl ester monomers, ${ }^{\left[{ }^{[0]}\right.}$ and chymotrypsin-cleavable monomers. ${ }^{[10]}$ With these chemistries, stimuli and/or controlled degradation could be obtained. The groups of Zhao ${ }^{[11]}$ and Bowman ${ }^{[12]}$ reported the 3D printing of linear polymers as a way to impart reprintability or recyclability to the printed object.

Nevertheless, such materials have a lower chemical resistance due to the polymer solubility in organic solvent. Another possibility to induce recycling of cross-linked 3D objects could be to use the concept of dynamic covalent networks ${ }^{[13]}$ also known as vitrimers. Such approach in combination with 3D printing has been already investigated. ${ }^{[14-16]}$ In contrary to these techniques the polymer ablation of conventional resins has received less attention, ${ }^{[17-18]}$ since this technique is more difficult to achieve and seems less promising. 

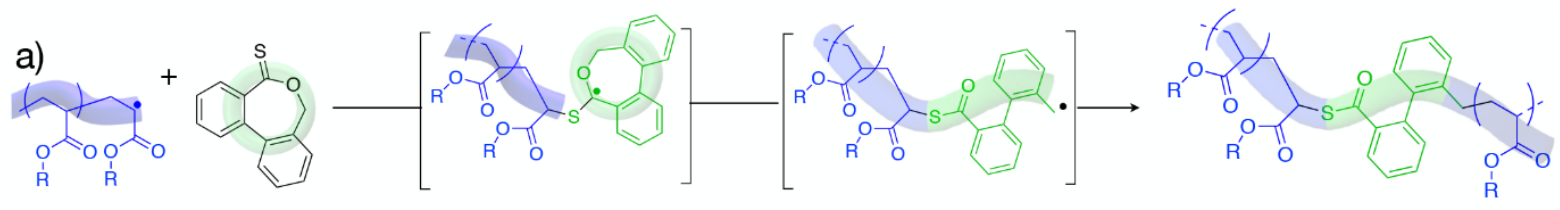

b)

Degradable materials via the cleavage of the knots

Degradable materials via the cleavage of the backbone
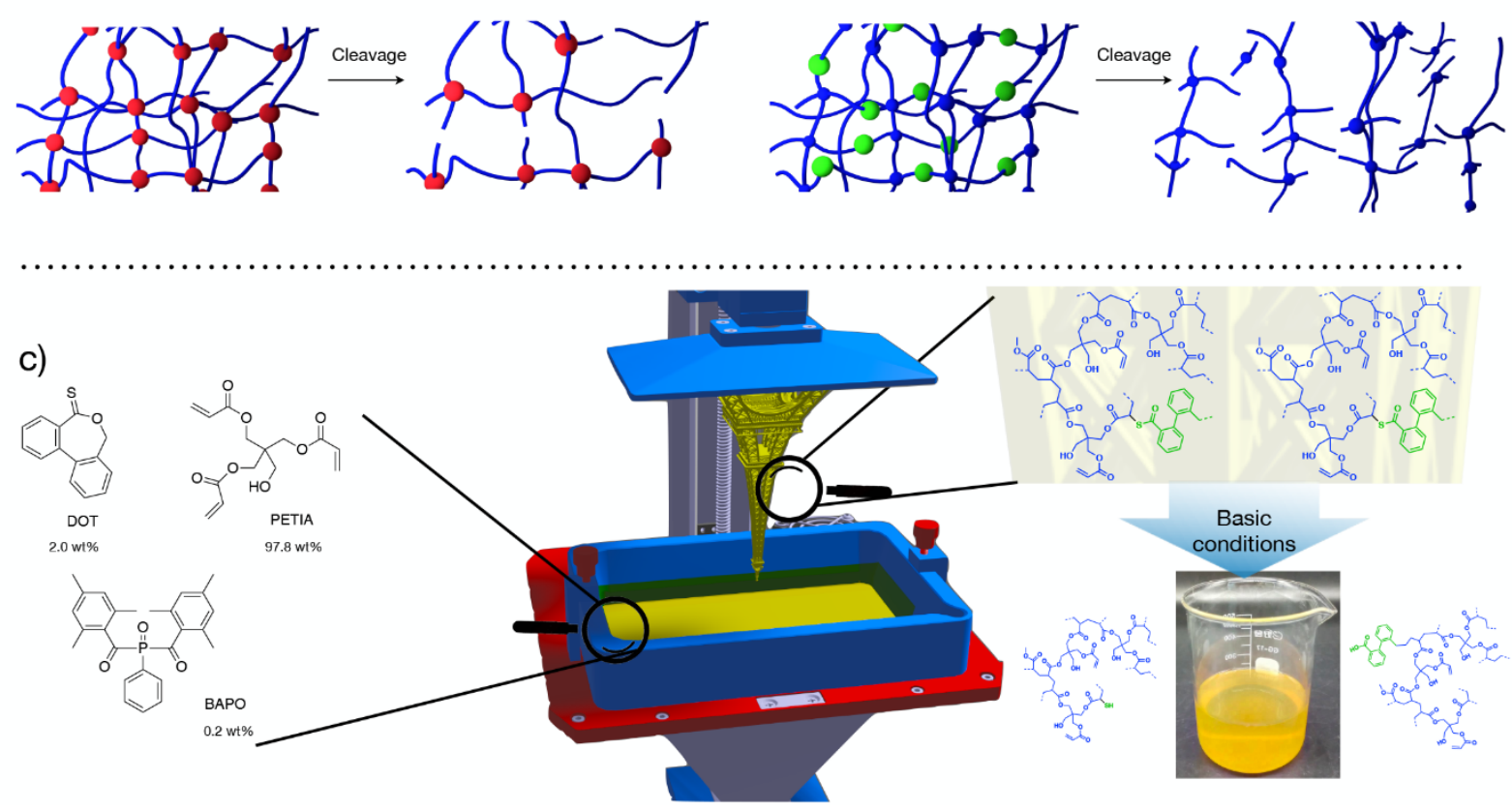

Figure 1. Schematic representation of the writing/erasing process. a) structure and mechanism of the radical polymerization involving the DOT/acrylate monomer pair. b) efficient cleavage of the thermoset via the comonomer approach (cleavage of 10 bonds in the case of both cleavable knots or cleavage linkage in the polymer backbone) adapted from ref. [19], c) Resin formulation, preparation of 3D objects via VAT 3D printer and cleavage mechanism.

All the previously mentioned techniques either require to develop new monomers/chemistries and thus cannot be readily used (due to the high quantity of monomers that has to be prepared for each $3 \mathrm{D}$ printing) on commercially-available resins/printers. Herein we thus introduce a new approach to impart degradability of 3D objects by adding a comonomer, here the dibenzo[c,e]-oxepane5 -thione (DOT) as an additive (2 $\mathrm{wt} \%$ ) to impart degradability to conventional acrylate resin (Figure 1.a). This approach is also based on the comonomer approach that has been developed by Johnson et al. ${ }^{[19-20]}$ on thermoset made by Ring-Opening Methathesis Polymerization (ROMP), where he showed that if the labile/degradable linkage is located into the backbone instead of the knots, fewer amount of labile/degradable moieties is required (Figure 1.b). This study showed that the cleavable monomer approach with only a few wt\% of additive is possible, ${ }^{[20]}$ leading to materials with similar mechanical properties but that could be turned soluble after degradation.

Radical ring-opening polymerization (rROP) is a technique that has been developed in the 80 s and that allows the incorporation of heteroatoms in the polymer backbone via a mechanism of addition/fragmentation. ${ }^{[21]}$ This technique has been rejuvenated recently by the copolymerization of common vinyl monomers and such cyclic derivatives to impart (bio)degradation of conventional materials. ${ }^{[21]}$ Two main cyclic monomer families emerged, the cyclic ketene acetals (CKAs) ${ }^{[21-22]}$ and the thionolactones ${ }^{[23-24]}$. The non-favorable reactivity ratio between CKA and

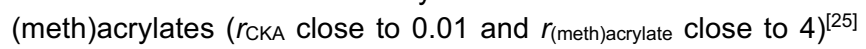
led to a low and non-homogeneous incorporation of the labile group in the materials. ${ }^{[26]}$ To impart good degradability, high initial amounts of CKA are required or a continuous feed of monomers ${ }^{[27]}$ could be used to counterbalance it. In the case of (meth)acrylate resin for 3D printing, both solutions cannot be implemented since high rate of polymerization is required implying using CKA only as additive and only bath resin can be used in $3 D$ printer. As a reference, preliminary tests were performed using CKA inserted in (meth)acrylate resin and do not lead to any degradation (see Table S1 for details).

Unlike CKAs, thionolactones and in particular dibenzo[c,e]oxepane-5-thione (DOT) introduced by Roth and coworkers ${ }^{[23]}$ followed by Gutekunst et al. ${ }^{[24]}$ presented an efficient copolymerization behavior (statistical incorporation of the two monomers) with acrylate derivatives, imparting homogeneous degradation that could occur via either hydrolysis or aminolysis (Figure 1.c). The combination of DOT/acrylate polymerization and VAT photopolymerization could be an efficient way to prepare (bio)degradable 3D objects. It has been shown that only few percents (5-10 vol\%) of cleavable units are required to impart degradability of thermosets ${ }^{[19-20]}$ and also that DOT has a slightly 
higher rate of incorporation ${ }^{[24]}$ than acrylate derivatives during their copolymerization, allowing to use a very low amount of DOT as additive.

\section{Results and Discussion}

Resin formulations were prepared by mixing only $2 \mathrm{wt} \%$ of DOT with pentaerythritol triacrylate (PETIA) as model multi-acrylate monomer and $0.2-0.5 \mathrm{wt} \%$ of bis(2,4,6-trimethylbenzoyl)-phenyl phosphine oxide (BAPO) as photoinitiator. Figure 2.a compares the absorption spectra of the resins, with and without DOT. The monomer chosen is transparent in UV $(\lambda>350 \mathrm{~nm})$ and the photoinitiator was chosen for its absorption at the wavelength of interest in this work (365-385 nm), both for laser microstructuring and for the DLP printer. DOT, added at $2 \mathrm{wt} \%$, shows significant absorption in the UV and visible $(<500 \mathrm{~nm})$ range. This absorption corresponds to a yellow coloration of the resin and is due to the $(\mathrm{C}=\mathrm{S})-\mathrm{O}$ - group. Once polymerized and due to the isomerization (Figure 1.a), the resulting ring-opened $(\mathrm{C}=\mathrm{O})-\mathrm{S}$ - thioester bond loose its color. It should thus be noted that this coloration also decreases strongly after UV irradiation (Figure 2.a). Nevertheless, since the overall conversion is not complete during the printing, the 3D objects are still yellow. The absorption of the DOT slightly modifies the reactivity of the resin but does not alter too much the polymerization rate. It has to be reminded that UV resins for 3D printing usually comprise photoabsorbers to control the penetration depth of actinic light. The kinetics of the

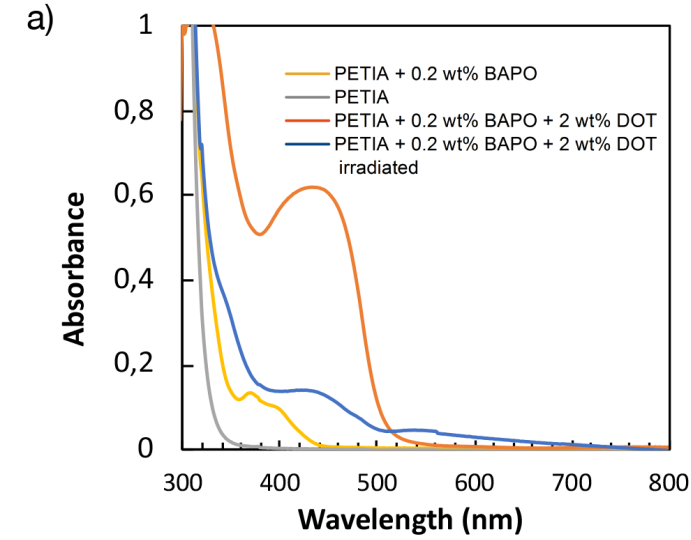

c)

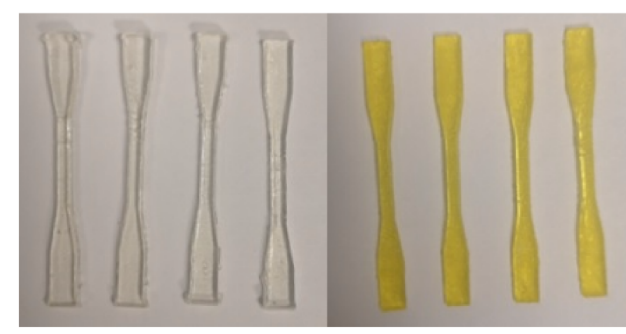

photopolymerization were studied to quantify the effect of the DOT on the photopolymerization process. The kinetics are plotted in Figure 2.b. They are based on monitoring the $\mathrm{C}=\mathrm{C}$ conversion of the acrylate functions in the monomer. With a BAPO concentration of $0.5 \mathrm{wt} \%$, the polymerization is efficient, leading to a final conversion of more than $60 \%$ and a conversion plateau quickly reached. This type of kinetics is classical for such a triacrylate monomer.

The trifunctional character ensures a very fast initial polymerization speed, then the rapid increase in viscosity leads to an important gelification of the medium which stops the polymerization and prevents a higher conversion. The addition of DOT (2 wt\%) decreases, as expected, the efficiency of the photopolymerization, both for the initial speed and the final conversion (50\%), but keep them still totally compatible to an 3D printing process. This effect can be easily explained by the absorption of DOT which acts as a filter effect for the BAPO, decreasing the amount of light available for photolysis of the photoinitiator. It should also be noted that a decrease of the kinetics during the copolymerization of acrylate derivatives and DOT is already observed during the thermal polymerization in solution. ${ }^{[23-24]}$ Finally, it can be observed that it is possible to reduce the concentration of BAPO to $0.2 \mathrm{wt} \%$, while maintaining effective polymerization, with in particular sufficient cross-linking of the polymer to confer interesting mechanical properties to the material, as observed qualitatively after irradiation. This result justifies the percentage of $0.2 \mathrm{wt} \%$ of BAPO used in the following. b)

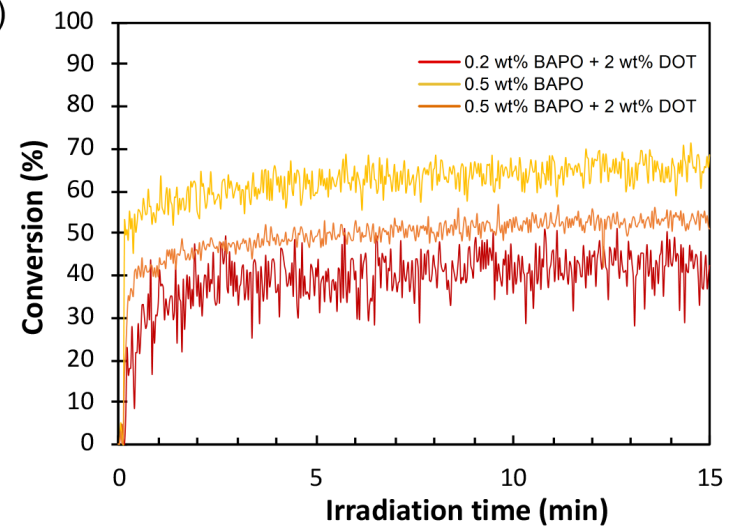

d)

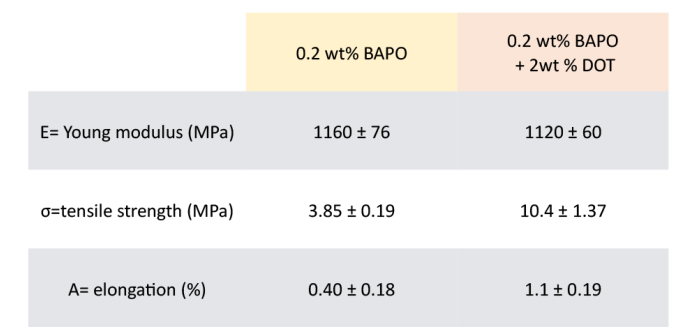

Figure 2. a) UV-vis absorption spectra of monomer (PETIA), monomer + photoinitiator (BAPO, $0.2 \mathrm{wt} \%$ ), monomer + photoinitiator + DOT (2 wt \%) before and after UV irradiation. b) Photopolymerization kinetics followed by FTIR (conversion of $\mathrm{C}=\mathrm{C}$ band at $1635 \mathrm{~cm}^{-1}$ ) under UV exposure for the photopolymer loaded or not with DOT (2 wt\%). c) Samples prepared by 3D-printing for mechanical properties testing. d) Comparison of main mechanical properties of material with and without DOT. 
Figure 2.c shows that it is possible to use DLP-UV to very easily fabricate polymer specimens that can be used to quantitatively evaluate the mechanical properties of the formed polymers. It can be seen that the addition of DOT only sligthly modifies the mechanical properties of the polymer by leading to a slightly softer and less brittle polymer (Table 2.d). This property can be correlated with the reduced photopolymerization efficiency induced by the addition of DOT. Nevertheless, Young modulus, tensile strength and elongation values remain in the same order of magnitude, meaning that DOT can be added up to $2 \mathrm{wt} \%$ without changing too significantly the mechanical properties of the polymerized material.

PETIA monomer is a reference monomer in microstructuring applications, $2 \mathrm{D}$ and $3 \mathrm{D} \cdot{ }^{[28-30]}$ It is shown in the following that the addition of DOT does not degrade the resolution and the possibility to use it for 2D and 3D stereolithography applications, at the micro and macroscopic scale. In a first approach, a laser microstructuring configuration is used to demonstrate the ability of the material to be used for laser-induced 3D microfabrication. It is a laser microstructuring configuration at the output of optical fibers that has demonstrated its interest for applications in photonics or sensors. ${ }^{[11-33]}$ Here, we use this configuration essentially as a microstructuring setup that allows to test a new material in this microstructuring context with respect to its reactivity and resolution.

The experimental procedure is described schematically in Figure 3.a. Further experimental details can be found in SI and reference [28]. Briefly, an optical fiber is cleaved and the resin is deposited at the cleaved end of the fiber. A $375 \mathrm{~nm}$ laser is coupled into the optical fiber and the light emerging from the fiber induces photocrosslinking in the volume located in the extension of the core of the optical fiber. After rinsing by immersion for a few seconds in ethanol, the microtip is obtained on the optical fiber. The photonic conditions (power, time), the geometrical and mechanical properties of the polymer formed allow to apprehend the reactivity of different photopolymers in this context of microfabrication. As shown in Figure 3.b, the microstructuring can be efficiently carried out using the DOT doped formulation. As expected after examination of the polymerization kinetics, the reaction is less efficient and the irradiation parameters need to be adjusted, by increasing the irradiation power and/or time (see SI for details).

a)

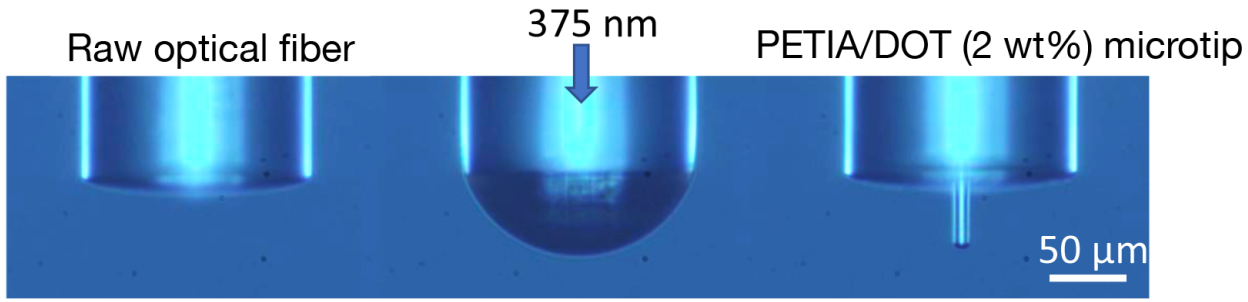

b)

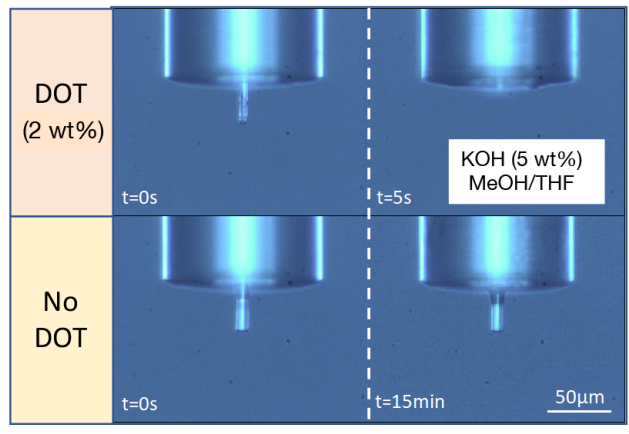

c)

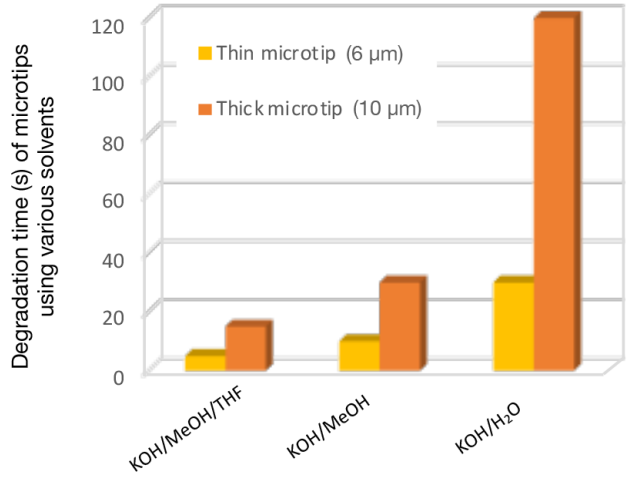

d)

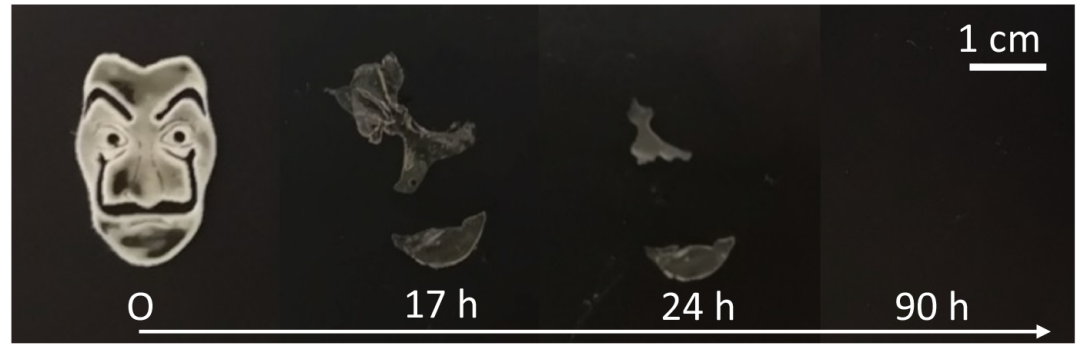

Immersion

Time using

$\mathrm{KOH}(5 \mathrm{wt} \%)$ in

$\mathrm{MeOH} / \mathrm{THF}$

Figure 3. a) Steps of process for preparing polymer microtips at the end of optical fiber showing the photopolymer drop deposition, exposure through the optical fiber and microtip after development (loaded with $2 \mathrm{wt} \%$ DOT in this case). b) Dissolution experiments with $\mathrm{KOH}(5 \mathrm{wt} \%)$ in MeOH/THF showing the efficiency of DOT on the dissolution kinetic of the microtip. c) comparison of degradation times for different solvents (in seconds), for thin and thick microtips (resp. 6 and 10 m diameter). d) Dissolution of a macroscopic object (resin PETIA + 2 wt\% DOT printed with a DLP-UV set-up) using $\mathrm{KOH}$ (5 wt\%) in MeOH/THF. 
From this result, we now consider the possibility of degrading the polymer after photopolymerization, as described schematically in Figure 1. To do this, we observe the behavior of microtips with or without DOT, in different solutions, a priori suitable to degrade the polymer.

First, a $\mathrm{KOH} / \mathrm{MeOH} / \mathrm{THF}$ mixture is used. In this mixture, $\mathrm{KOH}$ acts as a base for the hydrolysis of the polymer. The MeOH/THF mixture is selected to solubilize $\mathrm{KOH}$ and provide a solvent that can swell the polymer and thus promote its dissolution. As can be seen in Figure 3.b, the results after immersion are very different with and without DOT in the photopolymer: without DOT, the microtip remains attached to the end of the optical fiber after 15 min, showing the chemical resistance of this highly cross-linked polymer. With DOT in the resin, it is removed after only 5 seconds. Degradation is also obtained in different mixtures, as shown in Figure 3.c. $\mathrm{KOH} / \mathrm{MeOH}$ (without $\mathrm{THF}$ ) and $\mathrm{KOH}$ in water also degrade the microtip, but with less efficiency, as demonstrated by longer degradation times. Degradation is in any case longer for objects with various sizes, which is consistent with a degradation process as shown in Figure 1. At this stage, however, it is difficult to exclude a mechanism that would implement a detachment of the microtip from the fiber end by degradation of the polymer at the interface. The results shown in Figure 3.d confirm the degradation of the material in the volume. Indeed, in this experiment, a macroscopic object (Dali mask) was fabricated by DLP-UV. This object was then immersed in the $\mathrm{KOH} / \mathrm{MeOH} / \mathrm{THF}$ solution. After $17 \mathrm{~h}$, the object is taken out of the solution and placed on a substrate to be photographed. One can easily notice the already started degradation of the object. After $24 \mathrm{~h}$, this degradation is even more marked and finely, after $90 \mathrm{~h}$, the object is no longer visible in the solution. This result therefore undoubtedly confirms the role of DOT in the degradation mechanism. Very remarkably, a DOT concentration of only $2 \mathrm{wt} \%$ allows this property to be obtained. It seems quite logical that the degradation of macroscopic objects is significantly longer than that of microscopic objects. Moreover, the use of THF is important for these macroscopic objects to allow $\mathrm{KOH}$ access to hydrolysable functions.

Based on this result, we further demonstrate the possibility of manufacturing various objects by VAT photopolymerization. Different objects are manufactured, with different geometries, as shown in Figure 4.a and Figure 4.b using both setups, which are interesting to build 2.5 and $3 \mathrm{D}$ objects with arbitrary shapes. As can be seen, the residual yellow coloration is visible but the parts keep their transparency. a) DLP
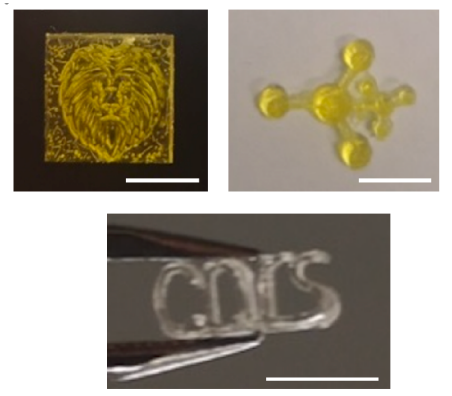

c) TPS

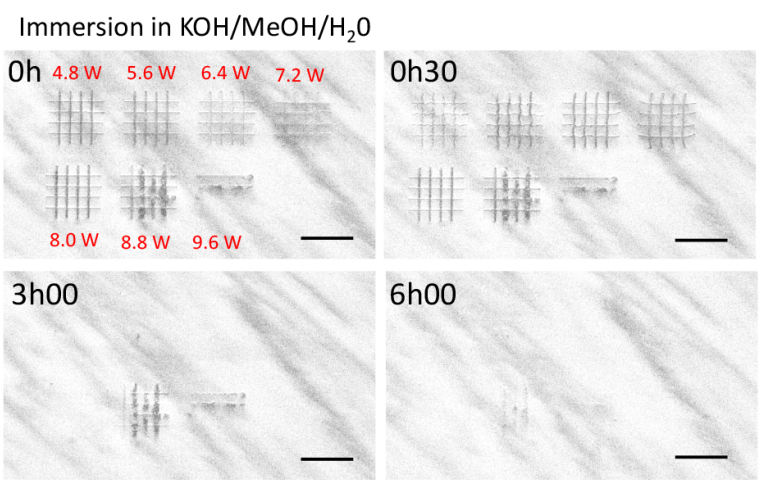

d) b) 3D printing

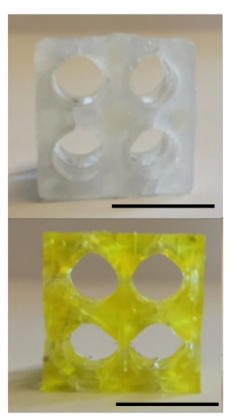

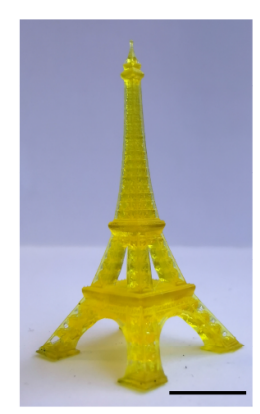

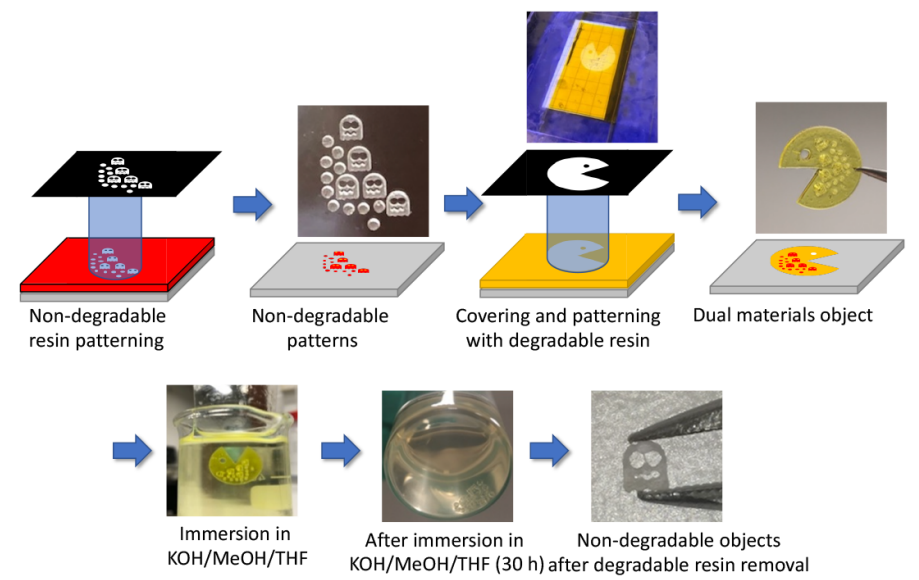

Figure 4. a) Examples of objects made by DLP-printing, b) 3D-printing (scale bar is $1 \mathrm{~cm}$ ) and c) Two-photon stereolithography of $50 \times 50$ mm microgrid structures and their dissolution in $\mathrm{KOH} / \mathrm{MeOH} / \mathrm{H}_{2} \mathrm{O}$. Grids were written using a 40X, 0.65-NA objective with increasing laser input power from 4.8 to 9.6 W. d) Embedding a non-degradable object in the degradable resin: example of a degradable Pacman head containing ghosts and balls that are non-degradable. 
For relatively thin objects such as the CNRS logo, the coloration can be attenuated after bleaching. Figure 4.c demonstrates that DOT-doped resin can also be used in two-photon stereolithography. To demonstrate this, micrometer grids (50x50 $\mu \mathrm{m})$ were produced with different laser power (irradiation wavelength $800 \mathrm{~nm}$ ). As for UV microfabrication, the resin doped with DOT was slightly less reactive but it could be used with photoreactivity in the same order of magnitude. A test of dissolution of the microgrids showed a complete degradation after 6 hours. Interestingly, it appeared that the dissolution time was increased when laser power was increased. This can be explained by a higher crosslinking of the polymer network at higher laser power. This proof of concept opens the doors towards 3D fabrication of arbitrary structures at the microscale.

The degradation property of PETIA/DOT combined with the stability of PETIA makes it possible to consider manufacturing objects with degradable and non-degradable parts using the same DLP-UV printer. This possibility is demonstrated in Figure 4.d. In this example, the strategy is as follows: in a first step, objects (ghosts and balls) are made with the non-degradable PETIA/BAPO resin. The object is then placed in the degradable resin with $2 \mathrm{wt} \%$ DOT and a second pattern is printed (Pacman figure). At the end, the object is a Pacman head in which objects are included. This matrix is soluble in the usual solution ( $5 \mathrm{wt} \%$ $\mathrm{KOH}$ in $\mathrm{MeOH} / \mathrm{THF}$ ) and its dissolution releases the included objects which are not degraded in the time necessary for the disappearance of the degradable matrix. This example is only intended to show the proof of concept of this new approach and the perspectives opened.

In this last section, we further explore the degradation conditions for 3D objects. Before the 3D printing, $\mathrm{cm}$-scale small tablets were prepared using a polypropylene mold previously printed by FDM (see Figure $\mathrm{S} 1$ for details) to find the best degradation conditions. The weight and visual aspect of these tablets were monitored during the decomposition process (Figure 5). According to the literature data, the degradation of the copolymers bearing thioesters weak bond could be done either via aminolysis using primary amines ${ }^{[23-24]}$ or using strong bases such as TBD or $\mathrm{KOH} / \mathrm{NaOH}$. We thus started the degradation at room temperature using a $50 \mathrm{vol} \%$ of $\mathrm{N}$-isopropylamine in dichloromethane, $2.5 \mathrm{wt} \%$ of TBD in THF and $5 \mathrm{wt} \%$ of $\mathrm{KOH}$ in $\mathrm{MeOH} / \mathrm{THF}$. We also prepared a pellet without DOT as a reference. After 3 months there is no degradation for both pellets using either $\mathrm{N}$-isopropylamine or TBD (see SI for details). This could be related to the difficult diffusion of such compounds into the dense PETIA-based 3D network. Nevertheless, the use of 5 wt $\%$ of $\mathrm{KOH}$ in $\mathrm{MeOH} / \mathrm{THF}$ led to the complete dissolution of the pellet in 1 week whereas the reference pellet was not degraded in 3 months (Figure 5a). To speed-up the degradation, we thus increase either the concentration of $\mathrm{KOH}$ or increase the temperature. Such kind of degradation conditions $\left(120{ }^{\circ} \mathrm{C}, 10\right.$ wt\% $\mathrm{KOH}$ in $\mathrm{EtOH}, 24 \mathrm{~h}$ ) were already used by Mecking et al. ${ }^{[34]}$ to perform the degradation of solid polyethylene objects containing weak ester linkages. Using these improved conditions, the complete dissolution of this dense $\mathrm{cm}$-scale object could be obtained in $17 \mathrm{~h}$ (Figure 5a).

The main goal of this study was the 3D printing of a degradable objects and we thus used our formulation (PETIA + $2 \mathrm{wt} \%$ DOT + $0.2 \mathrm{wt} \% \mathrm{BAPO}$ ) and the reference PETIA resin with a commercial $3 \mathrm{D}$ printer (Miicraft 125, $385 \mathrm{~nm}$ ). The complete parameters used for these printings are given in ESI and the same parameters were used for both resins. We first printed a hollow cube as a proof of concept. The printed objects are depicted in Figure $5 \mathrm{~b}$. It can be seen that the introduction of DOT inside the formulation do not modify the $3 \mathrm{D}$ printing since a similar object is obtained. Such cubes were then subjected to degradation. In this case, due to the porosity of the cube we choose to perform the degradation using $5 \mathrm{wt} \%$ of $\mathrm{KOH}$ in $\mathrm{MeOH} / \mathrm{THF}$ at room temperature and without agitation. After $8 \mathrm{~h}$ the hollow cube that was prepared with $2 \mathrm{wt} \%$ of DOT totally degraded (see the video in SI and Figure $5 \mathrm{~b}$ ) whereas the reference cube was not affected. This experiment validates the concept of degradable thermoset via the introduction of weak bond via radical ring-opening polymerization. These hollow cubes (the pristine PETIA and the DOT dopped cube) were also subjected to biodegradation using a home-made compost. Both cubes were inserted into the compost and the degradation monitored visually. The results are presented in Figure $5 \mathrm{c}$. After 20 days in the compost, the DOT-dopped cube is quite totally degraded whereas the PETIA one was not affected.

To further showcase the interest of our approach, an Eiffel Tower was also printed to ensure that a 3D object with a high resolution could be obtained and degraded. The results are gathered on Figure $6 \mathbf{a}$ and confirmed that this approach could be used on cm-scale 3D objects with a high resolution. The degradation of this Eiffel Tower presented an inhomogeneous degradation with the thinner part being degraded before the thicker ones. This feature is in good agreement with our previous findings correlating the rate of degradation with the size of the object. This prompt use to investigate the potential use of this approach to address the issue of support structures. Indeed, printing complex geometries with VAT photopolymerization usually require support structures that have to be manually removed afterward. In some cases, such structures are not easily accessible, and their removal could be difficult to perform or could be time consuming since it has to be done manually. Moreover, the preparation of easily removable/cleavable support structure could enable the printing of stacked 3D objects in the z-dimension. To illustrate this feature, we prepare two dices that are printed vertically (z-direction) using support structures (Figure 6b). The dices are printed either using the PETIA and the DOT-dopped resins. In both cases the 3D objects were obtained and were immersed in a gentle basic solution $(5 \mathrm{wt} \% \mathrm{KOH}$ in $\mathrm{MeOH}, \mathrm{RT}$, without agitation). After one hour, the dices printed with the DOT-dopped resin dissociated (Figure $6 \mathbf{b}$ ) whereas the reference still possessed its support structures. 
a)

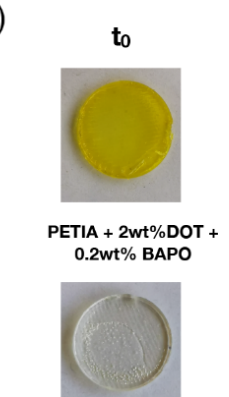

PETIA + 0.2wt $\%$ BAPO
KOH 5wt\% $\mathrm{MeOH} / \mathrm{THF}$ - RT

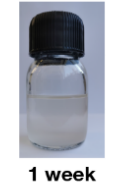

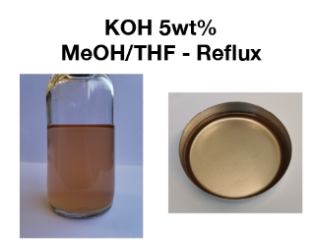

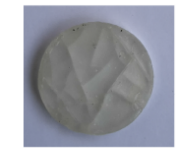

4 days
KOH 10wt $\%$

$\mathrm{MeOH} / \mathrm{THF}$ - Reflux
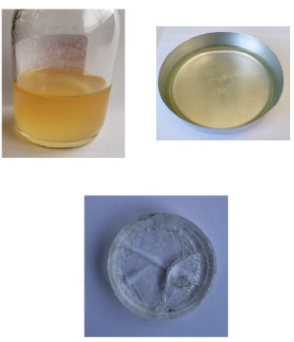

24 hours

b)

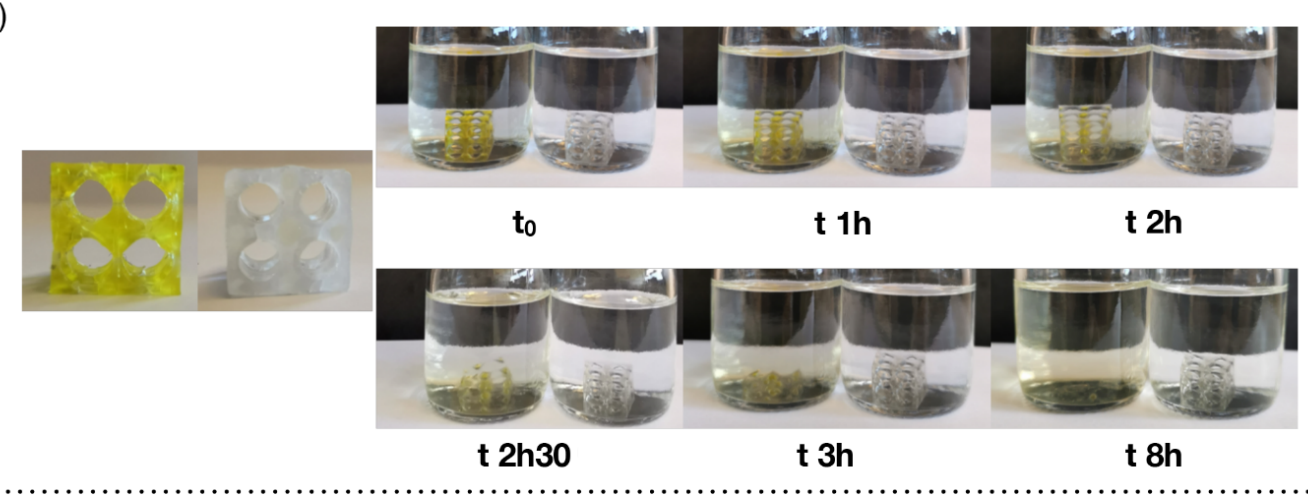

c)

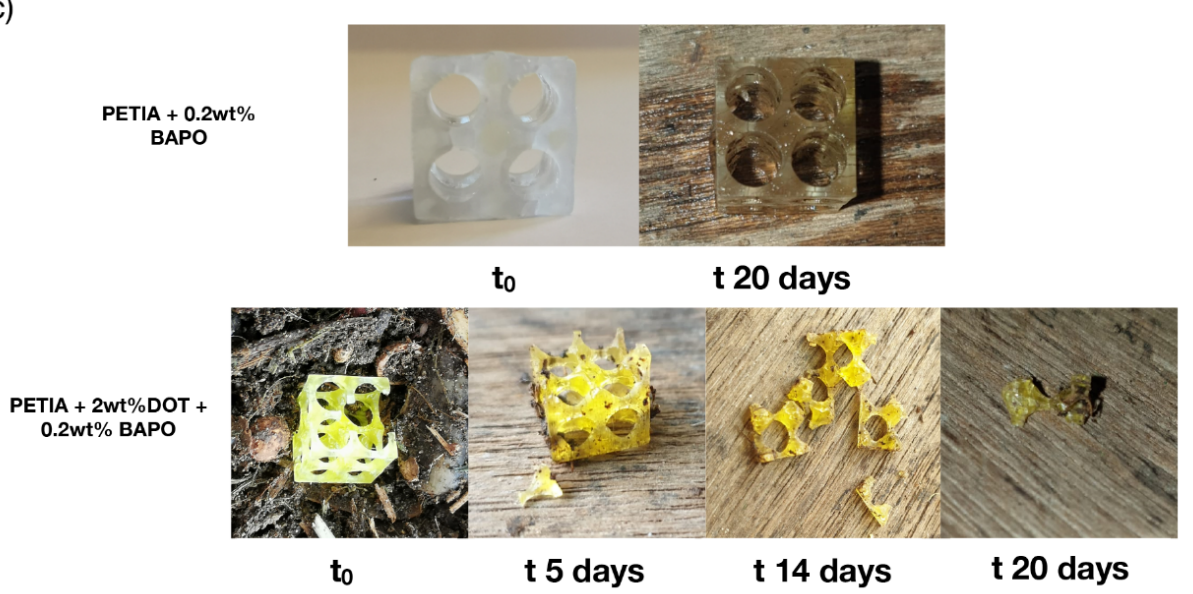

Figure 5. a). Degradation of the PETIA-based pellets using various degradation conditions. b) 3D printing of hollow cubes using a MiiCraft 125 UV-printer and their degradation ( $5 \mathrm{wt} \%$ of $\mathrm{KOH}$ in $\mathrm{MeOH} / \mathrm{THF}$ at room temperature and without agitation). c) Biodegradation of the hollow cubes in a home-made compost.

\section{Conclusion}

In conclusion, we showed in this work that the introduction of 2 wt $\%$ of a specific thionolactone, i.e. the dibenzo[c,e]-oxepane-5thione (DOT) that is known to copolymerize efficiently with acrylate monomers, into an acrylate based- $3 D$ resin confer a degradability property to the obtained 3D objects. Moreover, we showed that the mechanical properties of the final product are only slightly affected since only small amount of DOT was used as additive. This approach has then been applied to microstructuring configuration, two-photon stereolithography, DLP set-up and finally to commercial 3D printer. At all scales, the objects could be degraded using basic conditions. Such conditions are nevertheless dependent of the size of the object.
In all cases the complete dissolution of the objects occurred that allow first to strongly decrease the volume of wastes that could be produced via VAT photopolymerization and secondly will make more feasible the use of substractive manufacturing since only an additive could be added to common acrylate-based resins. As an example, using the size-dependent rate of degradation of the printed materials, stacked 3D object (z-directions) could be prepared and whose separation could be done gently in solution. This approach could thus be improved by developing new monomers of radical ring-opening polymerization that do not absorb in the UV range and whose reactivity with methacrylate derivatives is efficient (i.e. reactivity ratios allowing statistical incorporation of thioesters in the polymer backbone). 
a)

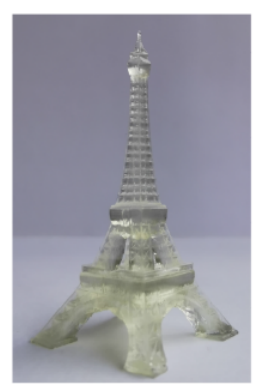

PETIA + 0.2wt \% BAPO

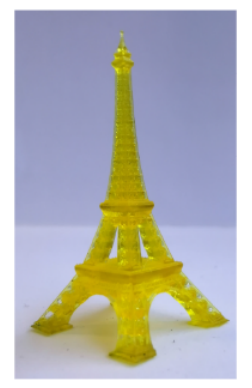

PETIA + 2wt \%DOT + $0.2 w t \%$ BAPO

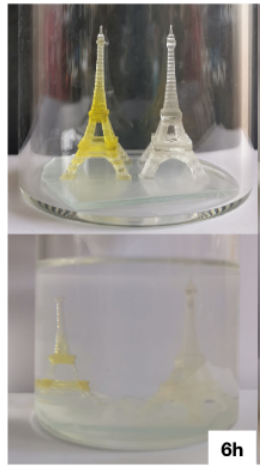

New solution

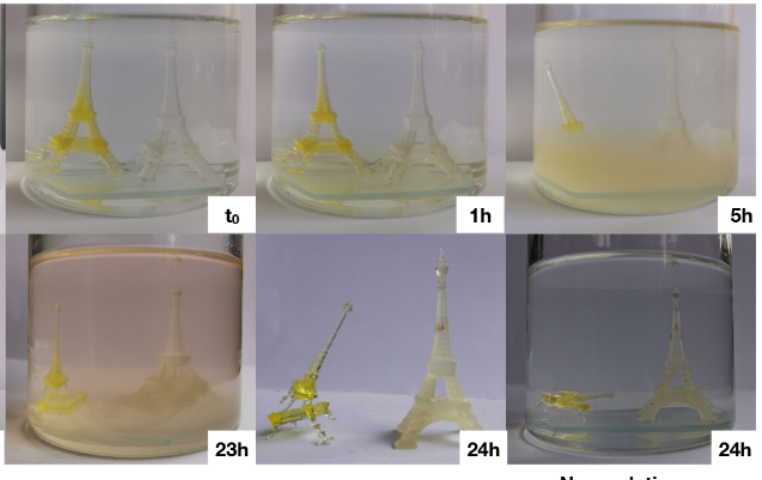

New solution

b)
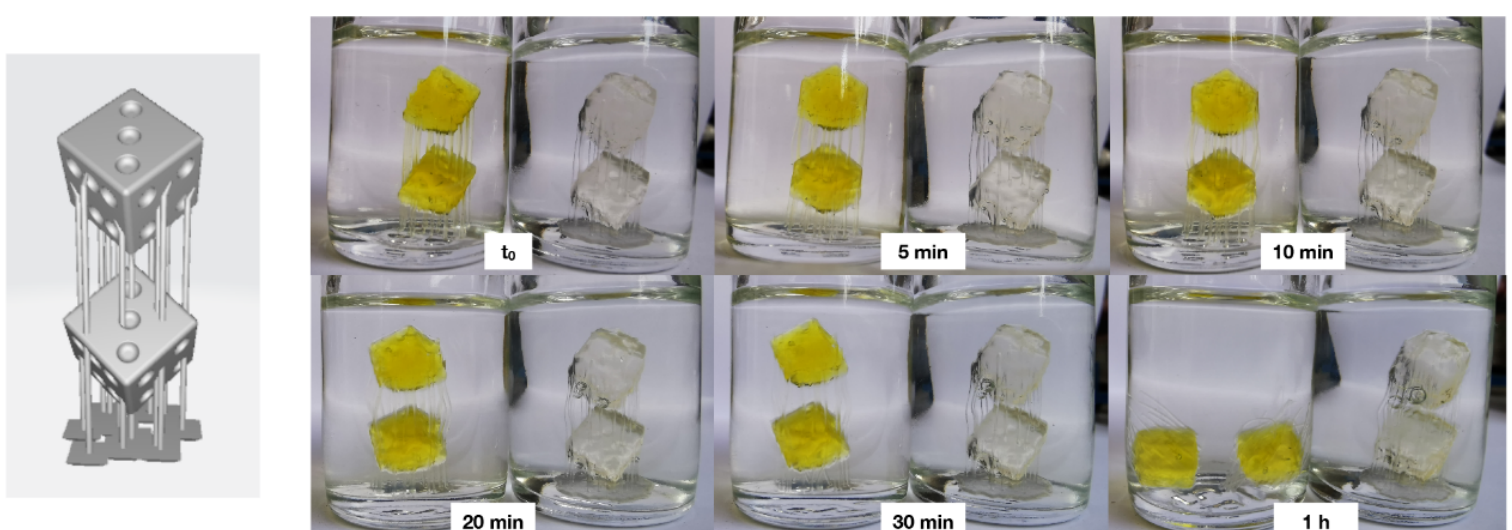

Figure 6. a) Preparation of the Eiffel Tower using the Miicraft 3D printer and its degradation using 5 wt $\%$ of $\mathrm{KOH}$ in $\mathrm{MeOH} / \mathrm{THF}$ at room temperature and without agitation. b) Preparation of stack dices using the Miicraft 3D printer and its degradation using $5 \mathrm{wt} \%$ of $\mathrm{KOH}$ in $\mathrm{MeOH} / \mathrm{THF}$ at room temperature and without agitation.

\section{Experimental Section}

Materials. All reagents and solvents were used as received Pentaerythritol triacrylate and isopropylamine was purchased from SigmaAldrich, Phenylbis(2,4,6-trimethylbenzoyl)phosphine oxide) (BAPO), Ammonia (4\% in $\mathrm{MeOH}, 2.0 \mathrm{~mol} / \mathrm{L}$ ) and 1,5,7-Triazabicyclo[4.4.0]dec-5ene (TBD) was purchased from TCI. SPLINT resin was purchased from Atom3D. Dibenzo[c,e]-oxepane-5-thione (DOT) was synthetized according to the literature procedure. ${ }^{[23]}$ 2-Methylene-1,3-Dioxepane (MDO) was synthetized according to the literature procedure. ${ }^{[35]}$

Resins preparation. Resin with CKA: 2 or 10 wt \% of MDO was mixing to SPLINT resin. The mixture was stirring during $1 \mathrm{~h}$ at room temperature away from light. For PETIA resin: $0.2 \mathrm{wt} \%$ of BAPO photoinitiator was mixing to triacrylate PETIA. The mixture was stirring during 3 days at room temperature away from the light. For PETIA/DOT resin: 2 wt $\%$ of DOT and $0.2 \mathrm{wt} \%$ of BAPO photoinitiator was mixing to triacrylate. The mixture was stirring during 3 days at room temperature away from the light.

Material characterization. The photopolymerization was monitored in situ by real-time Fourier transformed infrared spectroscopy with a ThermoNicolet 6700 IR-spectrometer. $\mathrm{KBr}$ pellets were used as substrates and a polypropylene film was used to avoid interaction with atmosphere. Irradiation was performed in situ using a $100 \mathrm{~W}$ Mercury-Xenon Lamp (LC 9588/02A from Hamamatsu) equipped with a band pass filter centred at $365 \mathrm{~nm}$ (A9616-07 from Hamamatsu). The conversion ratio was calculated from the decrease of the $1635 \mathrm{~cm}^{-1}$ band. The mechanical tests were performed with the INSTRON 4505 Dynamometer modernized ZWICK/ROELL (TestXpert II software).

Photofabrication Microstructuring on optical fiber was achieved on a home-made setup. For DLP microfabrication, a DLP projector (IkarusFullHD DLP6500light engine module from Invision) was used. 3D Printing was performed on a Miicraft 125 series from Miicraft. A mode-locked Ti: Sapphire oscillator was used as laser source (Chameleon Ultra II; pulse duration: $\sim 140 \mathrm{fs}$; repetition rate: $80 \mathrm{MHz}$ ) with a $\mu \mathrm{Fab}-3 \mathrm{D}$ system for microfabrication. More details of Two-Photon Microfabrication can be found in reference ${ }^{[36]}$.Grids with power ranging from $4.8 \mathrm{~W}$ to $9.6 \mathrm{~W}$ were written on glass slides and developed in ethanol.

\section{Acknowledgements}

We thank the CNRS for support and the French National Research Agency (ANR-15-CE08-0019 and ANR-19- CE190012) for the PhD funding of Noémie Gil and Constance Thomas. 
Keywords: 3D printing $\cdot$ degradable materials $\cdot$ radical ringopening polymerization, photopolymerization $\cdot$ microstructuring $\bullet$ thionolactone

[1] S. C. Ligon, R. Liska, J. Stampfl, M. Gurr, R. Mulhaupt, Chem Rev 2017, 117, 10212-10290.

[2] Z. Zhao, X. Tian, X. Song, Journal of Materials Chemistry C 2020, 8, 13896-13917.

[3] E. Sanchez-Rexach, T. G. Johnston, C. Jehanno, H Sardon, A. Nelson, Chemistry of Materials 2020, 32, 71057119.

[4] D. Grafe, S. L. Walden, J. Blinco, M. Wegener, E. Blasco, C. Barner-Kowollik, Angewandte Chemie-International Edition 2020, 59, 6330-6340.

[5] C. Barner-Kowollik, M. Bastmeyer, E. Blasco, G. Delaittre, P. Muller, B. Richter, M. Wegener, Angewandte ChemieInternational Edition 2017, 56, 15828-15845.

[6] M. M. Zieger, P. Müller, E. Blasco, C. Petit, V. Hahn, L. Michalek, H. Mutlu, M. Wegener, C. Barner-Kowollik, Advanced Functional Materials 2018, 28, 1801405.

[7] H. A. Houck, P. Muller, M. Wegener, C. Barner-Kowollik, F. E. Du Prez, E. Blasco, Adv Mater 2020, 32, e2003060.

[8] D. Grafe, A. Wickberg, M. M. Zieger, M. Wegener, E. Blasco, C. Barner-Kowollik, Nat Commun 2018, 9, 2788.

[9] R. Batchelor, T. Messer, M. Hippler, M. Wegener, C. Barner-Kowollik, E. Blasco, Adv Mater 2019, 31, e1904085

[10] D. Gräfe, M. Gernhardt, J. Ren, E. Blasco, M. Wegener, M. A. Woodruff, C. Barner - Kowollik, Advanced Functional Materials 2020, 2006998.

[11] G. Zhu, Y. Hou, J. Xu, N. Zhao, Advanced Functional Materials 2020, 2007173.

[12] M. D. Alim, K. K. Childress, N. J. Baugh, A. M. Martinez, A. Davenport, B. D. Fairbanks, M. K. McBride, B. T. Worrell, J. W. Stansbury, R. R. McLeod, C. N. Bowman, Materials Horizons 2020, 7, 835-842.

[13] N. J. Van Zee, R. Nicolaÿ, Progress in Polymer Science 2020, 104.

[14] L. L. Robinson, J. L. Self, A. D. Fusi, M. W. Bates, J. R. de Alaniz, C. J. Hawker, C. M. Bates, C. S. Sample, Acs Macro Letters 2021, 10, 857-863.

[15] Q. Shi, K. Yu, X. Kuang, X. Mu, C. K. Dunn, M. L. Dunn, T. Wang, H. Jerry Qi, Materials Horizons 2017, 4, 598-607.

[16] B. Zhang, K. Kowsari, A. Serjouei, M. L. Dunn, Q. Ge, Nat Commun 2018, 9, 1831.

[17] C. Liao, W. Anderson, F. Antaw, M. Trau, ACS Appl Mater Interfaces 2018, 10, 4315-4323.

[18] W. Xiong, Y. S. Zhou, X. N. He, Y. Gao, M. MahjouriSamani, L. Jiang, T. Baldacchini, Y. F. Lu, Light: Science \& Applications 2012, 1, e6-e6.

[19] K. E. L. Husted, P. Shieh, D. J. Lundberg, S. L. Kristufek, J. A. Johnson, Acs Macro Letters 2021, 10, 805-810.

[20] P. Shieh, W. Zhang, K. E. L. Husted, S. L. Kristufek, B. Xiong, D. J. Lundberg, J. Lem, D. Veysset, Y. Sun, K. A. Nelson, D. L. Plata, J. A. Johnson, Nature 2020, 583, 542547.

[21] A. Tardy, J. Nicolas, D. Gigmes, C. Lefay, Y. Guillaneuf, Chem. Rev. 2017, 117, 1319-1406.

[22] S. Agarwal, Polymer Chemistry 2010, 1, 953-964.

[23] N. M. Bingham, P. J. Roth, Chem Commun (Camb) 2018 , 55, 55-58.

[24] R. A. Smith, G. Fu, O. McAteer, M. Xu, W. R. Gutekunst, J Am Chem Soc 2019, 141, 1446-1451.

[25] J.-B. Lena, A. M. Van Herk, Industrial \& Engineering Chemistry Research 2019, 58, 20923-20931.

[26] D. Gigmes, P. H. M. Van Steenberge, D. Siri, D. R. D'Hooge, Y. Guillaneuf, C. Lefay, Macromolecular Rapid Communications 2018, 39, 1800193.

[27] J.-B. Lena, A. W. Jackson, L. R. Chennamaneni, C. T. Wong, F. Lim, Y. Andriani, P. Thoniyot, A. M. Van Herk, Macromolecules 2020, 53, 3994-4011.

[28] I. Dika, F. Diot, V. Bardinal, J. P. Malval, C. Ecoffet, A. Bruyant, D. Barat, B. Reig, J. B. Doucet, T. Camps, O.
Soppera, Journal of Polymer Science 2020, 58, 17961809.

[29] F. Kameche, W. Heni, S. Telitel, L. Vidal, S. Marguet, L. Douillard, C. Fiorini-Debuisschert, R. Bachelot, O. Soppera, Journal of Physical Chemistry C 2021, 125, 8719-8731.

[30] O. Soppera, S. Jradi, D. J. Lougnot, Journal of Polymer Science Part a-Polymer Chemistry 2008, 46, 3783-3794.

[31] P. A. S. Jorge, C. Maule, O. Soppera, P. V. S. Marques, leee Photonics Technology Letters 2011, 23, 492-494.

[32] S. Jradi, O. Soppera, D. J. Lougnot, Applied Optics 2008, 47, 3987-3993.

[33] O. Soppera, C. Turck, D. J. Lougnot, Optics Letters 2009, 34, 461-463.

[34] M. Haussler, M. Eck, D. Rothauer, S. Mecking, Nature 2021, 590, 423-427.

[35] A. Tardy, J.-C. Honoré, D. Siri, J. Nicolas, D. Gigmes, C. Lefay, Y. Guillaneuf, Polymer Chemistry 2017, 8, 51395147.

[36] M. Jin, J. Xie, J.-P. Malval, A. Spangenberg, O. Soppera, D.-L. Versace, T. Leclerc, H. Pan, D. Wan, H. Pu, P. Baldeck, O. Poizat, S. Knopf, J. Mater. Chem. C 2014, 2, 7201-7215. 


\section{Entry for the Table of Contents}

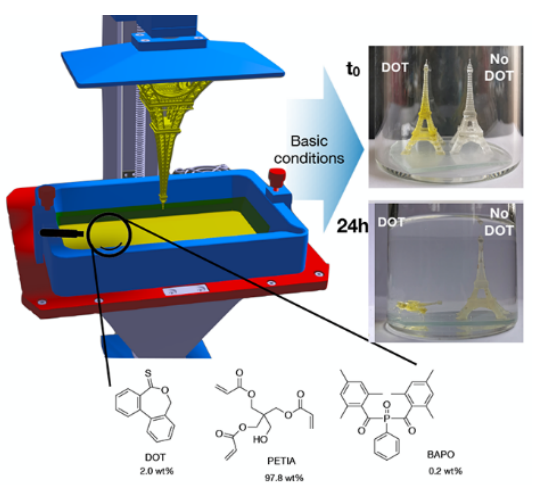

3D UV-printing produces cross-linked materials at the expense of degradability. The introduction of thionolactone ( $2 \mathrm{wt} \%$ of dibenzo[c,e]-oxepane-5-thione (DOT)) in acrylate-based resins confer (bio)degradability via the introduction of thioester units into the network via a radical ring-opening polymerization process. The resin was used in different set-up including commercial 3D printers and the objects were shown to degrade in basic solution.

Institute and/or researcher Twitter usernames: @IcrUmr7273, @YGuillaneuf 